\title{
ON NON-NEGATIVE SPECTRUM IN BANACH ALGEBRAS
}

\author{
by BERTRAM YOOD $\dagger$ \\ (Received 20th November 1972)
}

\section{Introduction}

Let $A$ be a complex Banach algebra with an identity 1 . In this note we study the subset $\Gamma$ of $A$ consisting of all $g \in A$ such that the spectrum of $g, s p(g)$, contains at least one non-negative real number. Clearly $\Gamma$ is not, in general, a semi-group with respect to either addition or multiplication. However, $\Gamma$ is an instance of a subset $Q$ of $A$ with the following properties, where $\rho(f)$ denotes the spectral radius of $f(4$, p. 30$)$.

(a) If $f \in Q$ and $\mathrm{t} \geqq 0$, then $t f \in Q$.

(b) If $f \in Q$ and $\rho(f)<1$, then $f(1-f)^{-1} \in Q$.

(c) The distance of $Q$ from -1 is larger than zero.

(d) If $f \in Q$ and $\rho(f)<1$, then $1-f \in Q$.

(e) If $f \in Q$ and $\rho(f)<1$, then $(1-f)^{-1} \in Q$.

(f) If $f \in Q$ and $t>0$, then $t+f \in Q$.

For our purpose (the characterisation of $\Gamma$ ) properties $(d),(e)$ and $(f)$ are not as useful as $(b)$. To see this consider the Banach algebra $B$ of all complexvalued functions on the compact Hausdorff space $E$ in the sup norm. The subset $Q$ of all $f \in B$ where $\operatorname{Re} f(t) \geqq 0$, for all $t \in E$, is a closed subset of $B$ satisfying properties $(a),(c),(d),(e)$ and $(f)$. Here $Q$ neither contains nor is contained in $\Gamma$. On the other hand, we shall see that $Q \subset \Gamma$ if $Q$ satisfies $(a),(b)$ and $(c)$.

Condition $(b)$ can be restated in the language of quasi-inverses $(4$, p. 16). For if $g^{\prime}$ denotes the quasi-inverse of $g$, then $f(1-f)^{-1}=-f^{\prime}$.

\section{On the properties (a), (b) and $\Gamma$}

Theorem 1. Let $Q$ be a subset of $A$ with properties (a) and (b). Then either $Q$ is contained in $\Gamma$ or -1 lies in the closure of $Q$.

Proof. Notice that, for a complex number $z,|z /(1-z)|<1$ if and only if $\operatorname{Re}(z)<\frac{1}{2}$. Now let $D_{n}$ be the closed disc in the complex plane with centre at $n /\left(n^{2}-1\right)$ and radius $1 /\left(n^{2}-1\right), n=2, \ldots$. Then, for these values of $n$,

$$
|z /(1-n z)|<1 \text { if and only if } z \notin D_{n} \text {. }
$$

$\dagger$ This research was supported in part by the National Science Foundation.

E.M.S. $-18 / 4-D$ 
In these terms we define a set $G$ in the complex plane by

$$
G=\{z:|z|<1 \text { and }|z /(1-n z)|<1, \quad n=1,2, \ldots\}
$$

and can readily picture $G$ graphically. Observe that if $z \in G$ then $z /(1-z) \in G$.

We suppose that -1 is not in the closure of $Q$ and must show that, for each $f \in Q, \operatorname{sp}(f)$ contains a non-negative number.

To this end we show first that if $g \in Q$ and $s p(g) \subset G$, then $g$ has no inverse in $A$. We define by induction a sequence $\left\{g_{n}\right\}$ starting with $g_{1}=g(1-g)^{-1}$. Note that $g_{1} \in A$ and $s p\left(g_{1}\right) \subset G$. Then, setting $g_{n+1}=g_{n}\left(1-g_{n}\right)^{-1}$, we see that every $g_{n} \in Q$ and $s p\left(g_{n}\right) \subset G$. We show, by induction, that $(1-n g)^{-1}$ exists in $A$ and $g_{n}=g(1-n g)^{-1}, n=1,2, \ldots$ This is certainly true for $n=1$. Assuming this fact for $n$ we consider

Then

$$
1-g_{n}=[1-(n+1) g](1-n g)^{-1}
$$

$$
1-(n+1) g=\left(1-g_{n}\right)(1-n g)
$$

is the product of two invertible elements. Moreover, by (1), and the induction hypothesis, we get

$$
g_{n+1}=g(1-n g)^{-1}\left(1-g_{n}\right)^{-1}=g(1-(n+1) g)^{-1}
$$

Now that we have $g(1-n g)^{-1} \in Q$ for each positive integer $n$ we use (a) to get

$$
n g_{n}=g\left(n^{-1}-g\right)^{-1} \in Q
$$

If $g^{-1} \in A$, then, from (2), we see that -1 is in the closure of $Q$. Therefore $\mathrm{g}^{-1}$ fails to exist, as claimed.

Next let $f \in Q$. Suppose that $s p(f)$ is disjoint with $[0, \infty]$. As $s p(f)$ is compact there is a number $\alpha, 0<\alpha<\pi / 2$, so that $s p(f)$ is disjoint with the wedge $W$ of complex numbers of the form $z=r \exp (i \theta),-\alpha \leqq \theta \leqq \alpha$ and $0 \leqq r<\infty$. Moreover, $s p(a f)$ is disjoint with $W$ for all a $>0$.

Elementary computations show that $D_{n}$ is contained in the interior of $W$ for all $n=2,3, \ldots$ such that $n>\csc (\alpha)$. Let $N$ be the smallest of these integers. Note that $|z| \geqq(n+1)^{-1}$ for all $z \in D_{n}$. Therefore, if we choose $b>0$ so that $\|b f\|<(N+1)^{-1}$, we see that $s p(b f)$ is also disjoint with $D_{j}, j \leqq N$. This ensures that $s p(b f) \subset G$. But then, as shown above, $f^{-1}$ does not exist or $0 \in s p(f)$. This contradicts our assumption that $[0, \infty]$ is disjoint with $s p(f)$ and completes the proof.

Suppose $g \notin \Gamma$. Since $s p(g)$ is compact there is an open set $V$ in the complex plane containing $s p(g)$ and disjoint with $[0, \infty)$. By (4, Theorem 1.6.16), $s p(h) \subset V$ if $h \in A$ is sufficiently close to $g$. Consequently $\Gamma$ is closed in $A$ and we deduce the following result from our theorem.

Corollary 1. $\Gamma$ is the unique maximal element in the collection of closed subsets of $A$ with the properties (a), (b) and $(c)$.

These results also hold for a real Banach algebra $A$ as can be seen by considering the complexification (4) of $A$. 
Following Bonsall (1) (see also 2) we call a subset $B$ of $A$ a semi-algebra if, whenever $f, g \in B$ and $t$ is a non-negative scalar, we have $f+g \in B, f g \in B$ and tf $\in B$.

Corollary 2. Any closed semi-algebra $B$ in $A$ either contains -1 or is contained in $\Gamma$.

Proof. Let $f \in B, \rho(f)<1$. Then, inasmuch as

$$
f(1-f)^{-1}=\sum_{n=1}^{\infty} f^{n}
$$

we see that $f(1-f)^{-1} \in B$. Hence $B$ satisfies $(b)$ and Theorem 1 applies. Corollary 2 was obtained in an entirely different way by Civin and White (3, p. 242).

Bonsall (1) and Brown (2) study type 0 semi-algebras (semi-algebras $B$ which have the additional property that $(1+f)^{-1} \in B$ whenever $\left.f \in B\right)$. In this case $B$ has the following property.

$$
s p(f) \cap(-\infty, 0) \text { is void for each } f \in B .
$$

Property (3) is related to our earlier properties.

Proposition. Let $Q$ be any subset of $A$ with properties (a) and (b). Let $J$ be the set of all $g \in Q$ such that $s p(g)$ intersects $(-\infty, 0)$ vacuously. Then $J$ has properties $(a)$ and $(b)$.

Proof. Consider $g \in J$ with $\rho(g)<1$ and let $A_{0}$ be a maximal closed subalgebra of $A$ containing $f$. We let $\Phi$ denote the carrier space of $A_{0}$ and use (4, Theorem 1.6.14).

Set $h=g(1-g)^{-1}$. If there exists some $\lambda,-\infty<\lambda<0, \lambda \in s p(h)$ then, for some $\phi \in \Phi$ we have also

$$
\hat{g}(\phi) /(1-\hat{g}(\phi))=\lambda .
$$

From this we get $\hat{g}(\phi)=\lambda-\lambda \hat{g}(\phi)$. We cannot have $\lambda=-1$. If $\lambda<-1$, then

$$
\hat{g}(\phi)=\lambda(1+\lambda)>1
$$

contrary to $\rho(g)<1$. If $-1<\lambda<0$ we get $g(\phi)<0$ contrary to $g \in J$. Therefore $h \in J$.

\section{REFERENCES}

(1) F. F. Bonsall, Semi-algebras of continuous functions, Proc. London Math. Soc. (3) 10 (1960), 122-140.

(2) G. Brown, Type 0 semi-algebras in Banach algebras, J. London Math. Soc. 43 (1968), 482-486. 
(3) P. Civin and C. C. White, Maximal closed pre-primes in Banach algebras, Trans. Amer. Math. Soc. 147 (1970), 241-260.

(4) C. E. RickART, General Theory of Banach Algrbras (Van Nostrand, 1960).

Pennsylvania State University

UNIVERSITY PARK, PA. 16802 U.S.A. 